\title{
ATENCIÓN PRIMARIA DE LA SALUD: UN COMPROMISO PENDIENTE
}

\author{
PRIMARY HEALTH CARE: A PENDING COMMITMENT \\ Lely Solari ${ }^{1, a}$ \\ La atención primaria de salud es el cuidado esencial de la salud basado en métodos y tecnología \\ práctica, con sólida base científica y socialmente aceptable, que sea accesible a todos los individuos \\ y familias de la comunidad, a través de su total participación y a un costo que la comunidad y el país \\ puedan solventar y mantener en cada etapa de su desarrollo, con el espíritu de la autodeterminación.
}

Alma Ata, 1978

En el 2010, con motivo de un curso internacional, le pregunté a Pekka Puska, el expresidente de la Federación Mundial del Corazón y actual presidente de la Asociación Internacional de Institutos Nacionales de Salud Pública (IANPHI) cuál era la fórmula mágica por la cual Finlandia tenía uno de los mejores sistemas de salud del mundo. Estaba segura de prever la respuesta: el respeto a las regulaciones, aunado a un elevado producto bruto interno e índice de desarrollo humano de la población, a una excelente infraestructura en los establecimientos de salud desde el primer nivel de atención y un elevado nivel de entrenamiento de su personal de salud (reflejado en un Instituto Nacional de Salud en el que el 40\% de sus 1000 trabajadores son PhD), eran para mí razones más que suficientes. Me miró con asombro ante la absurda pregunta y me respondió lo que para él era obvio: Llevamos décadas priorizando la atención primaria de salud.

Pero, ¿a qué se refería concretamente Puska? Al leer la definición de atención primaria de la salud (APS) incluida en la declaración de Alma Ata, uno puede destacar las palabras "tecnología", "sólida base científica", "costo que se pueda solventar". Desde este punto de vista se puede considerar que, aunque con deficiencias importantes, se han dado diversos avances en nuestro país. Inicialmente, los programas de control y en la actualidad las "estrategias sanitarias", han venido implementando intervenciones de salud que cumplen con estas características. A los tratamientos gratuitos para malaria, tuberculosis y VIH/SIDA, la implementación del parto en los establecimientos de salud, las intensas campañas de vacunación infantil y las intervenciones nutricionales en niños viviendo en la pobreza, últimamente se han venido añadiendo el manejo de enfermedades crónicas no transmisibles, mentales y oncológicas. El Ministerio de Salud tiene amplia experiencia en la gestión e implementación de intervenciones como estas, y actualmente, en coordinación con el Ministerio de Economía y Finanzas, está trabajando en ordenar y expandir su financiamiento mediante los programas presupuestales, siguiendo la línea del "financiamiento por resultados". Estos programas basan sus intervenciones en la "mejor evidencia científica disponible", y se planea ir progresivamente extendiéndolos a todas las regiones de nuestro país. Al respecto, consideramos sumamente adecuado utilizar la ciencia para definir las actividades de salud ${ }^{(1)}$.

Sin embargo, desde otro punto de vista, podemos enfatizar otros elementos de la definición de APS, como "cuidado esencial", "accesible a todos", "total participación", "en cada etapa". Esto se refiere más bien a acercar las actividades de salud a la población, involucrarla activamente en su ejecución y, por encima de todo, considerar a todo peruano y peruana en el proceso, sea cual sea el grupo poblacional, cultural, y geográfico en el que se encuentre. Se refiere a un cuidado de la salud descentralizado, difundido, incorporado a la comunidad, adaptado a sus costumbres, enfocado en la prevención, integrado a otros sectores que también influyen en la salud de las poblaciones. Considero que en la actualidad nadie puede argumentar que hayamos dado pasos importantes en este aspecto, aunque iniciativas no han faltado. El Modelo de Atención Integral por Etapas de Vida del Ministerio de Salud ${ }^{(2)}$ y las Unidades Básicas de Atención Primaria de Salud de ESSALUD ${ }^{(3)}$, son evidencia de que el tema ha sido abordado. Sin embargo, la APS no puede ser manejada como un programa más y tampoco debe ser confundida con atención de salud en el nivel primario. La atención primaria de la salud debe ser la base

Editora científica, RPMESP

Médica infectóloga, MSc, PhD (c)

Recibido: 24-04-13 Aprobado: 08-05-13

Citar como: Solari L. Atención primaria de salud: un compromiso pendiente [editorial]. Rev Peru Med Exp Salud Publica. 2013;30(2):173-4. 
del sistema de salud, debe estar insertada a todo servicio de salud y toda intervención que se brinde a la población y se debe practicar en todos los niveles de complejidad, priorizando obviamente los más accesibles a la población.

¿Qué tienen en común una niña que muere por rabia en Amazonas, una mujer de la capital con escasos recursos que llega al hospital con cáncer de cuello uterino en estadio terminal y un agricultor de la sierra hemipléjico a consecuencia de hipertensión arterial? Que todas son condiciones totalmente prevenibles, que todas ocurren en grupos poblacionales excluidos de los sistemas de salud que existen en el país y que todas tienen un enorme impacto en años de vida perdidos, en productividad, y en costos monetarios y personales a las familias. En suma, todas estas personas tienen en común ser el triste reflejo de la inoperancia de la atención primaria de la salud en nuestro país. Por más intervenciones de probada efectividad que existan para manejar tempranamente las condiciones mencionadas y prevenir sus complicaciones, e independientemente de cuánto sustento científico hayan demostrado tener, si estas se canalizan a través de establecimientos de salud con inadecuado equipamiento, con trabajadores de salud sobrecargados laboralmente y pobremente incentivados y entrenados, sin la activa participación de la comunidad, la eficacia de estas será subóptima o nula ${ }^{(4)}$. El pobre nivel de avance en el ámbito de atención primaria de la salud en el Perú constituye una triste paradoja para el país que vio nacer a un pionero mundial de su concepto e implementación. El legado de Manuel Núñez Butrón llama a nuestra población a "despertar" y a usar "tanto agua y jabón como lápiz y papel" (5). Estamos a tiempo de promover un cambio de rumbo. Pero este cambio debe ser sistémico, como la atención que esperamos brindar. Así como nuestro fracaso en atención primaria no se explica únicamente por brechas de financiamiento, nuestros esfuerzos no deben estar orientados exclusivamente hacia conseguirlo. Países de escasos recursos con sólidos sistemas de APS han logrado indicadores de salud de países ricos, siendo el innegable ejemplo cubano uno de los mejor documentados ${ }^{(6)}$.
A nosotros nos esperan retos difíciles, aunque no imposibles de superar. En primer lugar, la adecuada capacitación y descentralización de nuestros recursos humanos en salud, a través de políticas intensivas de incentivos laborales (no solo monetarias, sino de vivienda y educación) requerirá de nuestra creatividad y capacidad de innovar. Adicionalmente, lograr una estrecha coordinación con otros sectores involucrados en los determinantes de la salud, como educación, transporte y medioambiente, a nivel central, regional, y distrital, demandará sólidos mecanismos de articulación y asegurar que los procesos regulatorios estén libres de intereses que pudieran obstaculizar políticas en pro de la salud. Y lo más importante será lograr que la comunidad participe activamente en su salud, lo cual comienza por ganarse su confianza, pasa por escuchar, respetar y atender a sus inquietudes e incluye educarla y lograr que asuma su responsabilidad. Constituye un fruto "colgando alto", de los que cuando se alcanzan, aunque sea en el largo plazo, dan enormes retornos.

Ayer fueron predominantemente las enfermedades infecciosas, en la actualidad las enfermedades crónicodegenerativas, las asociadas a la violencia, el cáncer. Sea cual sea la forma que asuma el reto a la salud de la comunidad, siempre necesitaremos de una primera línea de defensa eficaz, un sistema de salud sólido y enraizado en la comunidad para poder enfrentarlo. Un verdadero sistema de atención primaria de la salud. Ahora que tenemos más recursos disponibles, podemos decidir. Podemos reevaluar nuestro actual enfoque vertical y reorientarlo hacia la construcción de un sistema de salud basado en el fortalecimiento de una APS de calidad para todo peruano, organizando la concertada participación de todos los actores involucrados en su desarrollo e implementación. O podemos seguir alimentando nuestra desidia con el pretexto de que nuestros recursos, aunque crecientes, son insuficientes para solucionar todos los problemas de salud que nos aquejan, pensar que tenemos la salud que nos merecemos y esperar a que otros países construyan modelos exitosos para poder copiarlos. Es una decisión que nuestras autoridades de estado y sobre todo de salud no pueden seguir dejando pendiente. $Y$ es también un compromiso moral.

\section{REFERENCIAS BIBLIOGRÁFICAS}

1. Meessen B, Soucat A, Sekabaraga C. Performance-based financing: just a donor fad or a catalyst towards comprehensive health-care reform? Bull World Health Organ. 2011;89(2):153-6.

2. Perú, Ministerio de Salud. Modelo de atención integral en salud basado en familia y comunidad. Lima: MINSA; 2009.

3. Seguro Social de Salud. Atención Primaria [Internet]. Lima: EsSalud; c2013 [citado el
15 de marzo de 2013]. Disponible en: http:// www.essalud.gob.pe/atencion-primaria/

4. Huamán-Espino L, Aparco JP, Nuñez-Robles E, Gonzáles E, Pillaca J, Mayta-Tristán P. Consumo de suplementos con multimicronutrientes Chispitas y anemia en niños de 6 a 35 meses: estudio transversal en el contexto de una intervención poblacional en Apurímac, Perú. Rev Peru Med Exp Salud Publica. 2012;29(3):314-23.
5. Neyra J. Manuel Núñez Butrón. Rev Peru Med Exp Salud Publica. 2005;22(2):148-9.

6. Dresang LT, Brebrick L, Murray D, Shallue A, Sullivan-Vedder L. Family medicine in Cuba: community-oriented primary care and complementary and alternative medicine. J Am Board Fam Pract. 2005;18(4):297-303. 